\title{
FINITE SAMPLE IMPROVEMENTS IN STATISTICAL INFERENCE WITH I(1) PROCESSES ${ }^{\star}$
}

\author{
by \\ D Marinucci \\ Università la Sapienza, Rome \\ and \\ P M Robinson \\ London School of Economics and Political Science
}

Contents:

Abstract

1. Introduction

2. Fully-modified Frequency Domain Least Squares

3. Monte Carlo Evidence

References

Appendix

Tables I - VIII

The Suntory Centre

Suntory and Toyota International Centres for Economics and Related Disciplines

Discussion Paper

London School of Economics and Political Science

No.EM/01/422

Houghton Street

July 2001

London WC2A 2AE

Tel.: 020-7955 6698

The first author's research was supported by MURST and by ESRC Grant R000235892. The second author's research was supported by a Leverhulme Trust Personal Research Professorship and ESRC Grant R000235892. We are grateful to four referees for comments which have significantly improved the paper. 


\begin{abstract}
Robinson and Marinucci (1998) investigated the asymptotic behaviour of a narrow-band semiparametric procedure termed Frequency Domain Least Squares (FDLS) in the broad context of fractional cointegration analysis. Here we restrict to the standard case when the data are $/(1)$ and the cointegrating errors are $I(0)$, proving that modifications of the Fully-Modified Ordinary Least Squares (FM-OLS) procedure of Phillips and Hansen (1990) which use the FDLS idea have the same asymptotically desirable properties as FM-OLS, and, on the basis of a Monte Carlo study, find evidence that they have superior finite-sample properties; the new procedures are also shown to compare satisfactorily with parametric estimates.
\end{abstract}

Keywords: Fully-modified ordinary least squares; finite sample improvements; statistical inference with /(1) processes; Monte Carlo study; parametric estimates.

JEL No.: C22

C) by the authors. All rights reserved. Short sections of text, not to exceed two paragraphs, may be quoted without explicit permission provided that full credit, including (C) notice, is given to the source.

Contact address: Professor Peter M Robinson, Department of Economics, London School of Economics and Political Science, Houghton Street, London WC2A 2AE, e-mail: pm.robinson@lse.ac.uk 


\section{INTRODUCTION}

The contention that jointly dependent macroeconomic series often have unit roots, and may be cointegrated, has considerably influenced econometric research in recent years. Consider the model ("cointegrated system") for the jointly dependent $p_{1} \times 1$ and $p_{2} \times 1$ vector of observables $y_{t}$ and $x_{t}$, respectively,

$$
y_{t}=\Pi x_{t}+u_{1 t}, t \geq 1, x_{t}=x_{t-1}+u_{2 t}, t \geq 1, x_{0}=0
$$

where $\Pi$ is an unknown $p_{1} \times p_{2}$ matrix, and $u_{t}^{\prime}=\left(u_{1 t}^{\prime}, u_{2 t}^{\prime}\right)$ is a $p \times 1 I(0)$ vector sequence, that is, it is covariance stationary with zero mean and has a spectral density matrix that is bounded and nonsingular at the origin, with $p=p_{1}+p_{2} ; z_{t}^{\prime}=\left(y_{t}^{\prime}, x_{t}^{\prime}\right)$ are then said to be $I(1)$, and cointegrated of orders $(1,0)(C I(1,0))$; we do not assume $u_{1 t}$ to be uncorrelated with $u_{2 t}$ (or, hence, with $x_{t}$ ). Cointegrated systems like (1.1) have been considered in the analysis of various economic hypotheses, in the context of, for example, purchasing power parity and other models of exchange rate determination, present value models, life-cycle models of consumers' behaviour, and the quantity theory of money. In simultaneously modelling short- and long-run behaviour, deviations from cointegrating relationships can capture short-term adjustments, in the spirit of the Error-Correction Mechanism (ECM) representation of Sargan (1964) and Davidson et al. (1978). Inference on $I(1)$ cointegrated processes in the framework of a fully parametric ECM representation has been developed by, for example, Phillips (1991a) and Johansen $(1988,1995)$, where asymptotically optimal rules are presented, based on maximum likelihood estimation (MLE), and requiring a complete specification of the system, with standard chi-squared testing procedures justified asymptotically.

Complete specification is unnecessary, however, for the achievement of such desirable first-order asymptotic properties. Indeed semiparametric inference rules based on instrumental variables (see Sargan (1959)) were considered for the analysis of $I(1)$ 
processes by Phillips and Hansen (1990), who introduced a Fully Modified Ordinary Least Squares (FM-OLS) technique which is optimal in the context of nonparametric autocorrelation in $u_{t}$. Also, the efficient frequency domain approach originally introduced by Hannan (1963a,b), which adapts to disturbance autocorrelation of nonparametric form and permits inclusion of only a proper subset of frequencies, has been developed by Phillips (1991b) in the presence of unit roots.

A common thread of many such semiparametric procedures is their reliance on initial estimates of $\Pi$, typically by Ordinary Least Squares (OLS). In the far broader framework of fractional cointegration analysis, Robinson and Marinucci (1998) showed that the performance of OLS can be improved on in several circumstances, including the $C I(1,0)$ case, by a semiparametric narrow-band procedure termed Frequency Domain Least Squares (FDLS), originally suggested in the context of a stationary (bivariate) sequence $z_{t}$ with long memory by Robinson (1994); here, correlation between $x_{t}$ and $u_{1 t}$ in (1.1) renders OLS inconsistent due to simultaneous equation bias, but if $u_{1 t}$ has less memory than $x_{t}$ (for example, if it is $I(0)$ ), FDLS is consistent. This proposal was further developed in the stationary case by Robinson and Marinucci (1998), but especially when $z_{t}$, and possibly $u_{1 t}$, are nonstationary. Here, OLS is typically consistent if the memory in $x_{t}$ exceeds that of $u_{1 t}$, but, depending on the orders of integration of $x_{t}$ and $u_{1 t}$, FDLS can have the same limiting distributional behaviour as OLS, perhaps with less "second order bias", or even converges faster, indicating that the medium and high-frequency "information" discarded by FDLS is at best unimportant and at worst harmful. Our present case of $I(1) x_{t}$ and $I(0) u_{1 t}$ is one in which Robinson and Marinucci (1998) found FDLS to have less second-order bias than OLS, and corresponding finite-sample improvements in Monte Carlo simulations. However, both OLS and FDLS share the disadvantage of having a nonstandard limit distribution which is inconvenient for inference. The present paper combines the FM-OLS and FDLS ideas to provide estimates with the same desirable 
asymptotic properties as FM-OLS, but, according to our Monte Carlo investigation, superior finite-sample properties. Intuitively, the latter finding is due to the presence, to some higher-order, of simultaneous equation bias in FM-OLS, which is reduced by stressing a narrow band of frequencies around the origin.

The following section, after some discussion of theoretical background, reviews a modification of FM-OLS previously considered by Robinson and Marinucci (1998), introduces a "Fully Modified Frequency Domain Least Squares" procedure, and establishes its asymptotic behaviour. Section 3 investigates via a Monte Carlo experiment their finite-sample performance, comparing them with the more traditional FM-OLS and MLE procedures in terms of bias, standard deviation and distributional properties. We find the results for the new procedures to be encouraging.

In the sequel, we use $\Rightarrow$ to denote weak convergence, $\|$.$\| to denote Euclidean norm,$ and $C$ for a generic, positive constant; " $>$ " will be taken to signify positive definite when referring to matrices.

\section{FULLY-MODIFIED FREQUENCY DOMAIN LEAST SQUARES}

For the purpose of deriving asymptotical statistical properties we first introduce

Assumption A (1.1) holds, with $u_{t}=\Psi(L) \varepsilon_{t}, \Psi(L)=\sum_{j=0}^{\infty} \Psi_{j} L^{j}$, where $L$ is the lag operator, $\operatorname{det}\{\Psi(1)\} \neq 0, \sum_{j=0}^{\infty} j\left\|\Psi_{j}\right\|<\infty$, and $\left\{\varepsilon_{t}\right\}$ is a sequence of independent and identically distributed (i.i.d.) $p \times 1$ vectors such that $E \varepsilon_{t}=0, E \varepsilon_{t} \varepsilon_{t}^{\prime}=$ $\Sigma, \operatorname{det}\{\Sigma\} \neq 0, E\left\|\varepsilon_{t}\right\|^{4}<\infty$.

Assumption A covers a wide class of short memory linear processes, including stationary and invertible vector autoregressive moving averages driven by Gaussian 
innovations.

Let $B^{\prime}(r)=\left(B_{1}^{\prime}(r), B_{2}^{\prime}(r)\right)$ be $p$-dimensional Brownian motion with covariance matrix $\Omega=\Psi(1) \Sigma \Psi(1)^{\prime}$; under Assumption A, as $n \rightarrow \infty$,

$$
\begin{aligned}
n^{-2} \sum_{t=1}^{n}\left(x_{t}-\bar{x}\right)\left(x_{t}-\bar{x}\right)^{\prime} & \Rightarrow \int_{0}^{1} \bar{B}_{2}(r) \bar{B}_{2}(r)^{\prime} d r \\
n^{-1} \sum_{t=1}^{n} u_{1 t}\left(x_{t}-\bar{x}\right)^{\prime} & \Rightarrow \int_{0}^{1} d B_{1}(r) \bar{B}_{2}^{\prime}(r)+\Lambda_{12},
\end{aligned}
$$

see Phillips (1988), where $\bar{B}_{2}(r)=B_{2}(r)-\int_{0}^{1} B_{2}(r) d r, \Lambda_{12}=\sum_{j=0}^{\infty} \Gamma_{12}(j), \Gamma_{12}(j)=$ $E u_{1 j} u_{20}^{\prime}$ and $\bar{x}=n^{-1} \sum_{t=1}^{n} x_{t}$. Unless $\Omega$ is block diagonal, $B_{2}($.$) and B_{1}($.$) are not$ independent and the distributions of the right-hand sides of $(2.1) /(2.2)$ are nonstandard. Now define $\Omega_{12}=\sum_{j=-\infty}^{\infty} \Gamma_{12}(j), \Gamma_{22}(j)=E u_{2 j} u_{20}^{\prime}, \Omega_{22}=\sum_{j=-\infty}^{\infty} \Gamma_{22}(j)$ and $B_{1.2}(r)=B_{1}(r)-\Omega_{12} \Omega_{22}^{-1} B_{2}(r)$, the Gaussian process orthogonal to $B_{2}(r)$. The asymptotic distribution of the OLS estimates

$$
\widehat{\Pi}=\sum_{t=1}^{n} y_{t}\left(x_{t}-\bar{x}\right)^{\prime}\left\{\sum_{t=1}^{n}\left(x_{t}-\bar{x}\right)\left(x_{t}-\bar{x}\right)^{\prime}\right\}^{-1},
$$

as $n \rightarrow \infty$ is readily seen to be given by

$$
\begin{aligned}
n(\widehat{\Pi}-\Pi) & \Rightarrow\left\{\int_{0}^{1} d B_{1}(r) \bar{B}_{2}(r)+\Lambda_{12}\right\}\left\{\int_{0}^{1} \bar{B}_{2}(r) \bar{B}_{2}(r)^{\prime} d r\right\}^{-1} \\
& =A_{1}+A_{2}+A_{3}
\end{aligned}
$$

where

$$
\begin{aligned}
A_{1} & =\left\{\int_{0}^{1} d B_{1.2}(r) \bar{B}_{2}(r)^{\prime}\right\}\left\{\int_{0}^{1} \bar{B}_{2}(r) \bar{B}_{2}(r)^{\prime} d r\right\}^{-1} \\
& \equiv \int_{G>0} N\left(0, \Omega_{11.2} \otimes G\right) d P(G), G=\left\{\int_{0}^{1} \bar{B}_{2}(r) \bar{B}_{2}(r)^{\prime} d r\right\}^{-1} \\
A_{2} & =\Omega_{12} \Omega_{22}^{-1}\left\{\int_{0}^{1} d B_{2}(r) \bar{B}_{2}(r)^{\prime}\right\}\left\{\int_{0}^{1} \bar{B}_{2}(r) \bar{B}_{2}(r)^{\prime} d r\right\}^{-1} \\
A_{3} & =\Lambda_{12}\left\{\int_{0}^{1} \bar{B}_{2}(r) \bar{B}_{2}(r)^{\prime} d r\right\}^{-1},
\end{aligned}
$$


for $\Omega_{11.2}=\Omega_{11}-\Omega_{12} \Omega_{22}^{-1} \Omega_{21}$; hence $A_{1}$ is a median-unbiased mixture of normal distributions, $A_{2}$ is proportional to the "unit root distribution" (note $d B_{2}=d \bar{B}_{2}$ ) and $A_{3}$ is a second-order bias component. The $\Omega_{a b}, a, b=1,2$ and $\Lambda_{12}$ can be consistently estimated from

$$
\widehat{u}_{1 t}=y_{t}-\widehat{\Pi} x_{t}, \widehat{u}_{2 t}=u_{2 t}=x_{t}-x_{t-1}
$$

and using techniques from the rich statistical literature on nonparametric spectral density estimation (for a review see Robinson and Velasco (1997)). One type stressed in recent econometric literature is

$$
\widehat{\Omega}_{a b}=\sum_{j=-\ell}^{\ell} k\left(\frac{j}{\ell}\right) \widehat{\Gamma}_{a b}(j), \widehat{\Lambda}_{a b}=\sum_{j=0}^{\ell} k\left(\frac{j}{\ell}\right) \widehat{\Gamma}_{a b}(j),
$$

where for $a, b=1,2$,

$$
\widehat{\Gamma}_{a b}(j)=\frac{1}{n} \sum_{t=1}^{n-j} \widehat{u}_{a, t+j} \widehat{u}_{b, t}^{\prime} j \geq 0 ; \quad=\widehat{\Gamma}_{b a}(-j)^{\prime}, j<0,
$$

and we impose:

Assumption B The kernel function $k($.$) satisfies$

$$
k(.): R \rightarrow[-1,1], k(0)=1, k(x)=k(-x), \int_{-\infty}^{\infty} k^{2}(x) d x<\infty,
$$

and $k($.$) is continuous at 0$ and at all but at most finitely many other points, and the bandwidth sequence $\ell$ satisfies $\ell \rightarrow \infty, \ell=O\left(n^{1 / 2}\right)$ as $n \rightarrow \infty$.

Phillips and Hansen (1990) proposed a two-step estimate (FM-OLS) that eliminates $A_{2}$ and $A_{3}$ from (2.3),

$$
\widetilde{\Pi}_{F M} \stackrel{\text { def }}{=}\left\{\sum_{t=1}^{n} \widehat{y}_{t}^{+}\left(x_{t}-\bar{x}\right)^{\prime}-n \widehat{\delta}\right\}\left\{\sum_{t=1}^{n}\left(x_{t}-\bar{x}\right)\left(x_{t}-\bar{x}\right)^{\prime}\right\}^{-1},
$$


where

$$
\widehat{y}_{t}^{+}=y_{t}-\widehat{\Omega}_{12} \widehat{\Omega}_{22}^{-1} \widehat{u}_{2 t}, \widehat{\delta}=\widehat{\Lambda}_{12}-\widehat{\Omega}_{12} \widehat{\Omega}_{22}^{-1} \widehat{\Lambda}_{22}
$$

and established the convergence

$$
n\left(\widetilde{\Pi}_{F M}-\Pi\right) \Rightarrow A_{1} \text { as } n \rightarrow \infty
$$

Hence $\widetilde{\Pi}_{F M}$ belongs to the $L A M N$ (Locally Asymptotically Mixed Normal) family of distributions introduced by Jeganathan $(1980,1988)$, and as such it shares its nice asymptotic statistical properties: distributions are centred around zero, nuisance parameters involve only scale effects and can be easily eliminated for the purpose of inference, an optimal theory of inference applies (LeCam (1986)), and hypothesis testing can be conducted within the usual asymptotic chi-squared paradigm.

We now describe the FDLS procedure. For $\lambda_{j}=2 \pi j / n, j=1, \ldots, n-1$, we introduce the discrete Fourier transforms

$$
w_{x}\left(\lambda_{j}\right)=\frac{1}{\sqrt{2 \pi n}} \sum_{t=1}^{n} x_{t} \exp \left\{i \lambda_{j} t\right\}, w_{y}\left(\lambda_{j}\right)=\frac{1}{\sqrt{2 \pi n}} \sum_{t=1}^{n} y_{t} \exp \left\{i \lambda_{j} t\right\},
$$

and the periodogram and cross-periodogram matrices

$$
I_{x x}\left(\lambda_{j}\right)=w_{x}\left(\lambda_{j}\right) w_{x}\left(\lambda_{j}\right)^{*}, I_{y x}\left(\lambda_{j}\right)=w_{y}\left(\lambda_{j}\right) w_{x}\left(\lambda_{j}\right)^{*}
$$

the asterisk denoting transposition combined with complex conjugation. Also, we define the averaged periodogram matrices

$$
\widehat{F}_{x x}(1, m)=\frac{2 \pi}{n} \sum_{j=1}^{m} \operatorname{Re}\left\{I_{x x}\left(\lambda_{j}\right)\right\}, \widehat{F}_{y x}(1, m)=\frac{2 \pi}{n} \sum_{j=1}^{m} \operatorname{Re}\left\{I_{y x}\left(\lambda_{j}\right)\right\} .
$$

where $R e\{$.$\} denotes real part. The FDLS statistic is then defined for 1<m<n-1$ as

$$
\widehat{\Pi}_{m}=\widehat{F}_{y x}(1, m)\left\{\widehat{F}_{x x}(1, m)\right\}^{-1}
$$


(assuming the inverse exists), which for $m=n-1$ yields OLS (with intercept correction) $\widehat{\Pi}$, in view of Parseval's equality. We are interested, however, in the behaviour of FDLS under the bandwidth condition

$$
\frac{1}{m}+\frac{m}{n} \rightarrow 0, \text { as } n \rightarrow \infty
$$

which rules out OLS and moreover includes only a band of frequencies around zero that degenerates slowly as $n$ increases, as is required in spectral density estimation for stationary series. Phillips (1991b) proposed a system-type estimates of $\Pi$ based in effect on periodogram averages across a band of $m$ frequencies about zero satisfying (2.13), though his proofs actually pertain to weighted autocovariance estimates. We take $m<n / 2$, due to periodicity of period $\pi$, and evenness of the periodogram.

FDLS dominate OLS in several circumstances under (2.13), for example it is still consistent in cases where OLS is not, i.e., when $x_{t}$ and $u_{1 t}$ in (1.1) are stationary long memory processes, labelled the "stationary cointegration" case, and it exhibits a faster rate of convergence when $x_{t}, u_{1 t}$ have "less than unit-root-nonstationarity", i.e. when the collective memory in $x_{t}$ and $u_{1 t}$ in (1.1) is more limited than in the $C I(1,0)$ case, see Robinson and Marinucci (1998). In the more standard, unit root framework considered in this paper, Robinson and Marinucci (1998) established under Assumption A and (2.13) the convergence (see 2.3,2.4a,2.4b)

$$
n\left(\widehat{\Pi}_{m}-\Pi\right) \Rightarrow A_{1}+A_{2}
$$

because the second-order bias component

$$
\frac{m}{2 n} h(0)\left\{\widehat{F}_{x x}(1, m)\right\}^{-1} \rightarrow_{p} 0
$$

under (2.13), where

$$
h(0)=\frac{1}{2 \pi} \sum_{j=0}^{\infty}(2 j+1)\left\{\Gamma_{12}(j)-\Gamma_{12}(-j-1)\right\}
$$


is finite because Assumption A implies that

$$
\sum_{j=0}^{\infty} j\left\|\Gamma_{12}(j)\right\|<\infty .
$$

Thus FDLS has a the second-order bias of smaller order than that of OLS.

As in Robinson and Marinucci (1998), we consider a modification of FM-OLS (2.8), based on first step FDLS, rather than OLS, residuals, and denoted

$$
\begin{aligned}
\widetilde{\Pi}_{F M}^{*} & =\left\{\sum_{t=1}^{n} \widetilde{y}_{t}^{+}\left(x_{t}-\bar{x}\right)^{\prime}-n \widetilde{\delta}\right\}\left\{\sum_{t=1}^{n}\left(x_{t}-\bar{x}\right)\left(x_{t}-\bar{x}\right)^{\prime}\right\}^{-1} \\
\widetilde{y}_{t}^{+} & =y_{t}-\widetilde{\Omega}_{12} \widetilde{\Omega}_{22}^{-1} u_{2 t}, \widetilde{\delta}=\widetilde{\Lambda}_{12}-\widetilde{\Omega}_{12} \widetilde{\Omega}_{22}^{-1} \widetilde{\Lambda}_{22} \\
\widetilde{u}_{1 t} & =y_{t}-\widehat{\Pi}_{m} x_{t}, \widetilde{u}_{2 t}=u_{2 t}=x_{t}-x_{t-1}
\end{aligned}
$$

for $\widetilde{\Omega}_{a b}$ and $\widetilde{\Lambda}_{a b}, a, b=1,2$, defined as in $(2.6)$, (2.7) with $\widehat{\Gamma}_{a b}(j)$ replaced by $\widetilde{\Gamma}_{a b}(j)$ which employs the FDLS residuals (2.17) in place of $\widehat{u}_{a t}$ and $\widehat{u}_{b t}$.

A further alternative is to use the FDLS idea more directly, namely a narrowband version of FM-OLS which reproduces its capacity to achieve asymptotic mixed normality, Fully Modified Frequency Domain Least Squares (FM-FDLS):

$$
\widetilde{\Pi}_{F D}=\widehat{F}_{\widetilde{y}^{+} x}(1, m)\left\{\widehat{F}_{x x}(1, m)\right\}^{-1},
$$

where $\widehat{F}_{\widetilde{y}^{+} x}$ is defined analogously to $\widehat{F}_{y x}$ in $(2.11)$, with $y_{t}$ replaced by $\widetilde{y}_{t}^{+}$.

The estimates $\widetilde{\Pi}_{F M}^{*}$ and $\widetilde{\Pi}_{F D}$ share the same asymptotic distribution as $\widetilde{\Pi}_{F M}$, and are thus asymptotically optimal in the same sense. The proof of the following Theorem, which is given in the Appendix, relies on some general results on the asymptotic behaviour of the averaged (cross-) periodogram for nonstationary processes, see Robinson and Marinucci (1998, Proposition 4.2 and Lemma 5.4).

Theorem 1 Under Assumption A, B and (2.13), as $n \rightarrow \infty$

$$
n\left(\widetilde{\Pi}_{F M}^{*}-\Pi\right), n\left(\widetilde{\Pi}_{F D}-\Pi\right) \Rightarrow A_{1},
$$


where $A_{1}$ is given in $(2.4 \mathrm{a})$.

In view of Theorem 1 and (2.10), $\widetilde{\Pi}_{F M}, \widetilde{\Pi}_{F M}^{*}$, and $\widetilde{\Pi}_{F D}$ are asymptotically equivalent. However, because the latter two are all, in various related ways, less affected by second order bias, it seems natural to conjecture that these estimates may improve on $\widetilde{\Pi}_{F M}$ in finite samples. We now provide some Monte Carlo evidence to support this claim.

\section{MONTE CARLO EVIDENCE}

We start from the model of Gonzalo (1994) in Monte Carlo comparison of estimates of Johansen $(1988,1995)$ with simple (time-domain) estimates such as OLS:

$$
y_{t}=\Pi x_{t}+u_{1 t} \quad, x_{t}=\gamma y_{t}+w_{t} \quad, \quad w_{t}=w_{t-1}+e_{2 t} \quad, \quad x_{0}=0
$$

which is easily seen to be equivalent to (1.1) with $p_{1}=p_{2}=1$ and

$$
u_{2 t}=\frac{1}{1-\gamma \Pi}\left(\gamma\left(u_{1 t}-u_{1, t-1}\right)+e_{2 t}\right)
$$

We adopt two alternative specifications for $u_{1 t}$, namely

$$
\begin{aligned}
& \text { Model } A: \quad u_{1 t}=\rho u_{1, t-1}+e_{1 t} \\
& \text { Model } B: \quad u_{1 t}=\rho_{1} u_{1, t-1}+\rho_{2} u_{1, t-2}+e_{1 t}
\end{aligned}
$$

where $\left(e_{1 t}, e_{2 t}\right)^{\prime} \equiv i . i . d . N(0, \Sigma)$. Note that the consequent univariate specification of $u_{2 t}$ is $\operatorname{ARMA}(1,1)$ under Model $\mathrm{A}$ and $\operatorname{ARMA}(2,2)$ under Model $\mathrm{B}$, when $\gamma \neq 0$, and white noise when $\gamma=0$. We set

$$
\Pi=2, \quad \Sigma=\left[\begin{array}{ll}
1 & .5 \\
.5 & 1
\end{array}\right], \quad \rho_{2}=-.9
$$


and allow $\rho, \rho_{1}$ and $\gamma$ to vary, taking $\rho=.8, .4, .0,-.4,-.8, \rho_{1}=.947,34,-.34,-.947$, and $\gamma=1,0$. The spectral density of $u_{1 t}$ has a peak at zero frequency for $\rho>0$ and at $\pi$ for $\rho<0$ in Model $\mathrm{A}$, and at frequency $\arccos \left(-\rho_{1}\left(1+\rho_{2}\right) / 4 \rho_{2}\right)$ in Model B, that is at $\pi / 3,4 \pi / 9,5 \pi / 9$ and $2 \pi / 3$, respectively, for the four $\rho_{1}$. One expects the finite-sample performance of frequency domain procedures to be largely influenced by the locations and magnitudes of such peaks and by the exogeneity parameter $\gamma ; x_{t}$ is weakly exogenous if and only if $\gamma=0$.

The implementation of efficient estimation procedures on Models A and B requires the ECM representations

$$
\begin{aligned}
& \text { Model } A: \Delta z_{t}=\Psi_{A} z_{t-1}+\varepsilon_{A t} \\
& \text { Model } B: \Delta z_{t}=\Psi_{B} z_{t-1}+\Gamma \Delta z_{t-1}+\varepsilon_{B t}
\end{aligned}
$$

where $\Delta$ is the difference operator, $\varepsilon_{A t}, \varepsilon_{B t}$ are i.i.d. vectors and

$\Psi_{A}=(\rho-1)\left[\begin{array}{l}1 \\ \gamma\end{array}\right]\left[\begin{array}{c}1 \\ -\Pi\end{array}\right]^{\prime}, \Psi_{B}=\left(\rho_{1}+\rho_{2}-1\right)\left[\begin{array}{l}1 \\ \gamma\end{array}\right]\left[\begin{array}{c}1 \\ -\Pi\end{array}\right]^{\prime}, \Gamma=\rho_{2}\left[\begin{array}{l}1 \\ \gamma\end{array}\right]\left[\begin{array}{c}1 \\ -\Pi\end{array}\right]^{\prime}$.

These representation is the basis for implementing the estimate of Johansen (1988). His original procedure estimated a basis for the cointegrating space, rather than $\Pi$, but normalized estimates of $\Pi$, and their limiting distribution, can be readily obtained, see e.g. Johansen (1995, pp.179-184). For all series we estimated, by his procedure, both the equations

$$
\begin{aligned}
\Delta z_{t} & =\Upsilon\left[\begin{array}{ll}
1 & -\Pi
\end{array}\right] z_{t-1}+\varepsilon_{A t} \\
\Delta z_{t} & =\Upsilon\left[\begin{array}{ll}
1 & -\Pi
\end{array}\right] z_{t-1}+\Xi \Delta z_{t-1}+\varepsilon_{B t}
\end{aligned}
$$

where $\Upsilon$ is an unconstrained $2 \times 1$ vector and $\Xi$ is an unconstrained $2 \times 2$ matrix. By comparison with (3.1)-(3.3), it is apparent that (3.4) is just-identified with respect to Model A when $\gamma \neq 0$ but over-parameterized when $\gamma=0$, but is mis-specified with 
respect to Model B, whereas (3.5) is over-parameterized with respect to both Models A and B, the more so in case of Model A. We shall refer to Johansen's estimate as MLE but of course this description would only be accurate under just-identification.

The results, based on 5000 replications of series of lengths $n=64$ and 128, are summarized in Tables I through VIII; here $\widetilde{\Pi}_{F M}, \widetilde{\Pi}_{F M}^{*}$, and $\widetilde{\Pi}_{F D}$ are defined in (2.8), (2.16), and (2.18), respectively, and $\widetilde{\Pi}_{M 0}$ and $\widetilde{\Pi}_{M 1}$ are the MLEs based on (3.4), (3.5), respectively. We set the bandwidth parameter $\ell$ equal to the closest integer to $\sqrt{n}$, hence obtaining $\ell=8$ for $n=64$ and $\ell=11$ for $n=128$. We fixed $m=5$ for $n=64$ and $m=6$ for 128. (In Robinson and Marinucci (1998) the effect of varying $m$ was considered, in a different type of simulation experiment involving FDLS). Hence we have a total of $(2 \times 5 \times 2)+(2 \times 4 \times 2)=36$ groups of simulations for each estimate.

Tables I and II (Model A) and V and VI (Model B) illustrate our findings for Monte Carlo bias $(B)$ and standard deviation $(S D)$. A general feature of the results (which largely holds also in other tables) is a substantial overall improvement in performance of all estimates as $n$ increases, and considerably better results for $\gamma=0$ than for $\gamma=1$, with little difference between the various FM estimates when $\gamma=0$, but noticeable superiority in our narrow-band proposals over FM-OLS when $\gamma=1$. Another factor is the location of the spectral peak of $u_{1 t}$ (and thence of $u_{2 t}$ also). The "traditional" estimates $\widetilde{\Pi}_{F M}, \widetilde{\Pi}_{M 0}$ and $\widetilde{\Pi}_{M 1}$ are based upon the whole frequency band $[0, \pi]$, whereas $\widetilde{\Pi}_{F D}$ and, to a lesser extent, $\widetilde{\Pi}_{F M}^{*}$, focus on a degenerating band around the origin. One therefore expects our new proposals to perform better the further the spectral peak of $u_{1 t}$ is shifted away from the origin.

This is found indeed to be the case for both Model A and B. Using mean squared error $M S E=B^{2}+S D^{2}$ (not explicitly reported) as the basis for comparison, we find for Model A that $\widetilde{\Pi}_{M 0}$ is best in 13 cases out of $20, \widetilde{\Pi}_{F D}$ is best 4 times, and $\widetilde{\Pi}_{F M}^{*}$ thrice; remarkably, the "traditional" $\widetilde{\Pi}_{F M}$ estimate is dominated by at least one of the new procedures in all cases, often significantly, and this same finding also emerges 
when we examine $B$ and $S D$ separately. It is also noteworthy that $\widetilde{\Pi}_{F D}$ improves over $\widetilde{\Pi}_{M 1}$ in 11 cases, so that the loss here due to over-parameterization is greater than that due to our semiparametric aspect. A close inspection of (3.3) and (3.4) reveals that in the weakly exogenous case $\gamma=0$ the long-run behaviour derives from the equation for $y$ alone; irrespective of the super-consistency result, we could hence anticipate that limited information procedures such as the various FM estimators would be relatively efficient for Model A. We refer the reader to Johansen (1995) for an explanation of weak exogeneity in the VAR context. For Model B, the results are rather similar, but the MLE does better relatively speaking, bearing in mind the mis-specification in (3.6) and the over-parameterization in (3.7); $\widetilde{\Pi}_{M 1}$ is best in all 16 cases, and $\widetilde{\Pi}_{F D}$ is superior to $\widetilde{\Pi}_{M 0}$ in all but two. Note that although Johansen's procedure performs best in the vast majority of cases, it can produce very high $B$ and $S D$ in Model A for $\rho=0.8$. This phenomenon was previously noted by Gonzalo (1994, p. 217-219), and is due to the normalization which we adopt in order to estimate $\Pi$; in particular, $\widetilde{\Pi}_{M 0}$ and $\widetilde{\Pi}_{M 1}$ are ratios of random variables and need not have finite moments. Although in this sense our Monte Carlo study is somewhat unfair to MLE, for applied research the estimates of $\Pi$ in the triangular representation (1.1)-(1.3) are likely to be the most useful for testing economic hypotheses, at least in a bivariate context, see for instance the examples discussed by Hamilton (1994, p.651). When more than two variables are included, however, identification might hang on long-run causal relationships or restrictions on the cointegrating vector, which are not coherent to the triangular form we use here but to some observationally equivalent form.

In Tables III and IV (Model A) and VII and VIII (Model B) we report empirical sizes based on $\chi^{2}$ tests with nominal size $5 \%$,

$$
\chi_{\widetilde{\Pi}-\Pi}^{2}=\frac{(\widetilde{\Pi}-\Pi)^{2}}{\widehat{s}_{\widetilde{\Pi}}^{2} / \widehat{F}_{x x}(1, n-1)}, \widehat{s}_{\widetilde{\Pi}}^{2}=\frac{1}{n} \sum_{t=1}^{n}\left(y_{t}-\widetilde{\Pi} x_{t}\right)^{2}, \widetilde{\Pi}=\widetilde{\Pi}_{F M}, \widetilde{\Pi}_{F M}^{*}, \widetilde{\Pi}_{F D} ;
$$

for $\widetilde{\Pi}_{M 0}$ and $\widetilde{\Pi}_{M 1}$, we took the denominator in the $\chi^{2}$-statistics to be the $(2,2)$ - 
th element in the inverse of expression (13.12) on p.184 of Johansen (1995), which estimates their asymptotic variance. In terms of proximity to the nominal value, we find that $\widetilde{\Pi}_{F D}$ is best in 13 cases out of 20 in Model $\mathrm{A}, \widetilde{\Pi}_{F M}^{*}$ is best 4 times, and only in 3 cases is $\widetilde{\Pi}_{M 0}$ actually superior to the narrow-band procedures. Likewise, for Model B, $\widetilde{\Pi}_{F D}$ is best in 7 cases out of $16, \widetilde{\Pi}_{M 1}$ is best in 5 cases, $\widetilde{\Pi}_{M 0}$ in 3 and $\widetilde{\Pi}_{F M}$ in the remaining one.

In general, our "narrow-band" procedures, and in particular $\widetilde{\Pi}_{F D}$, emerge as promising competitors to traditional semiparametric estimates, at the same time providing a robust and efficient alternative to parametric methods even when the correct model is known, up to a few number of lags in the specification of the ECM, though it is unsurprising that the Johansen procedures tend to perform best overall in our experiment. One is likely to improve the finite-sample performance of each of the four semiparametric procedures by iteration (for example, obtaining new cointegrating residuals by FM-FDLS and then starting each procedure anew from the estimation of $\Omega$ and $\delta)$.

\section{REFERENCES}

Davidson, J.E.H., Hendry, D.F., Srba, F. and S. Yeo (1978) "Econometric Modelling of the Aggregate Time-Series Relationship Between Consumers' Expenditure and Income in the United Kingdom", Economic Journal, 88, 661-692

Gonzalo, J. (1994) "Five Alternative Methods of Estimating Long-Run Equilibrium Relationships", Journal of Econometrics, 60, 203-233

Hamilton, J.D. (1994) Time Series Analysis, Princeton University Press, Princeton

Hannan, E.J. (1963a) "Regression for Time Series" in M.Rosenblatt (Ed.) Time Series Analysis, J.Wiley, New York, 17-37 
Hannan, E.J. (1963b) "Regression for Time Series with Errors of Measurement", Biometrika, 50, 293-302

Hannan, E.J. (1970) Multiple Time Series, J.Wiley, New York

Jeganathan, P. (1980) "An Extension of a Result of LeCam Concerning Asymptotic Normality", Sankhya Series A, 42, 146-160

Jeganathan, P. (1988) "On the Strong Approximation of the Distributions of Estimators in Linear Stochastic Models, I and II: Stationary and Explosive Models", Annals of Statistics, 16, 1283-1314

Johansen, S. (1988) "Statistical Analysis of Cointegrating Vectors", Journal of Economic Dynamics and Control, 12, 231-254

Johansen, S. (1995) Likelihood-Based Inference in Cointegrated Vector AutoRegressive Models, Oxford University Press, New York

LeCam, L. (1986) Asymptotic Methods in Statistical Decision Theory, New York, J.Wiley

Phillips, P.C.B. (1988) "Weak Convergence of Sample Covariance Matrix to Stochastic Integrals via Martingale Approximation", Econometric Theory 4, 528-533

Phillips, P.C.B. (1991a) "Optimal Inference in Cointegrated Systems", Econometrica 59, 283-306

Phillips, P.C.B. (1991b) "Spectral Regression for Cointegrated Time Series", in W.A.Barnett, J.Powell and G.E.Tauchen (Eds.), A Nonparametric and Semiparametric Methods in Econometrics and Statistics, Cambridge, Cambridge University Press

Phillips, P.C.B. and B.E. Hansen (1990) "Statistical Inference in Instrumental Variables Regression with I(1) Variables", Review of Economic Studies, 53, 473-495

Robinson, P.M. (1994) "Semiparametric Analysis of Long Memory Time Series", Annals of Statistics, 22, 515-539

Robinson, P.M. and C. Velasco (1997) "Autocorrelation Robust Inference", 
in Handbook of Statistics, Volume 15, C.S. Maddala and C.R. Rao (Eds.), Elsevier Science Publisher BV, 267-298

Robinson, P.M. and D.Marinucci (1998) "Semiparametric Frequency Domain Analysis of Fractional Cointegration", STICERD Discussion Paper \#348

Sargan, J.D. (1959) "The Estimation of Relationships with Autocorrelated Residuals by the Use of Instrumental Variables", Journal of the Royal Statistical Society Series B, 21, 91-105

Sargan, J.D. (1964) "Wages and Prices in the United Kingdom: A Study in Econometric Methodology", in P.E. Hart, G. Mills, and J.K. Whitaker (Eds.), Econometric Analysis for National Economic Planning, Butterworth, London

\section{APPENDIX}

Proof of Theorem 1 In view of (2.10) it suffices to prove that

$$
\begin{aligned}
\left\|\widetilde{\Pi}_{F M}^{*}-\widetilde{\Pi}_{F M}\right\| & =o_{p}\left(n^{-1}\right), \\
n\left(\widetilde{\Pi}_{F D}-\Pi\right) & \Rightarrow A_{1} .
\end{aligned}
$$

We note that, as $n \rightarrow \infty$

$$
\widetilde{\Omega}_{22} \rightarrow_{p} \Omega_{22}, \widetilde{\Omega}_{12} \rightarrow_{p} \Omega_{12}, \widetilde{\delta} \rightarrow_{p} \delta
$$

by $n$ - consistency of $\widehat{\Pi}_{m}$ and standard manipulations. Thus the left-hand side of (A.1) is bounded in norm by

$$
\left\|\left(\widehat{\Omega}_{12} \widehat{\Omega}_{22}^{-1}-\widetilde{\Omega}_{12} \widetilde{\Omega}_{22}^{-1}\right) \sum_{t=1}^{n} u_{2 t}\left(x_{t}-\bar{x}\right)^{\prime}-n(\widetilde{\delta}-\widehat{\delta})\right\|\left\|\left\{\sum_{t=1}^{n}\left(x_{t}-\bar{x}\right)\left(x_{t}-\bar{x}\right)^{\prime}\right\}^{-1}\right\|,
$$

which is $o_{p}(n) O_{p}\left(n^{-2}\right)=o_{p}\left(n^{-1}\right)$, as desired, in view of (2.1), (2.2) and (A.4). For (A.2), consider

$$
y_{t}^{+}=y_{t}-\Omega_{12} \Omega_{22}^{-1} u_{2 t}=\Pi x_{t}+u_{1 t}-\Omega_{12} \Omega_{22}^{-1} u_{2 t},
$$


and introduce

$$
\widehat{\Pi}^{+}=\left\{\sum_{t=1}^{n} y_{t}^{+}\left(x_{t}-\bar{x}\right)^{\prime}\right\}\left\{\sum_{t=1}^{n}\left(x_{t}-\bar{x}\right)\left(x_{t}-\bar{x}\right)^{\prime}\right\}^{-1}
$$

from Phillips and Hansen (1990)

$$
n\left(\widehat{\Pi}^{+}-\Pi\right) \Rightarrow\left\{\int_{0}^{1} d B_{1.2}(r) \bar{B}_{2}(r)^{\prime}+\Lambda_{12}-\Omega_{12} \Omega_{22}^{-1} \Lambda_{22}\right\}\left\{\int_{0}^{1} \bar{B}_{2}(r) \bar{B}_{2}(r)^{\prime} d r\right\}^{-1} .
$$

In the same way we can define $\widetilde{\Pi}_{F D}^{+}=\widehat{F}_{y^{+} x}(1, m)\left\{\widehat{F}_{x x}(1, m)\right\}^{-1}$. Writing $u_{1.2, t}=$ $u_{1 t}-\Omega_{12} \Omega_{22}^{-1} u_{2 t}, \widehat{a}_{1.2}=\frac{1}{n} \sum_{t=1}^{n} u_{1.2, t}\left(x_{t}-\bar{x}\right)^{\prime}, \widetilde{a}_{1.2}=\widehat{F}_{u_{1.2}, x}(1, m)$, and applying the argument of Robinson and Marinucci (1998, Theorem 5.3), we find that

$$
\begin{aligned}
n\left(\widetilde{\Pi}_{F D}^{+}-\Pi\right) & =\left[\widehat{a}_{1.2}-E \widehat{a}_{1.2}+\left\{\left(\widetilde{a}_{1.2}-\widehat{a}_{1.2}\right)-E\left(\widetilde{a}_{1.2}-\widehat{a}_{1.2}\right)\right\}+E \widetilde{a}_{1.2}\right] \widehat{F}_{x x}(1, m) \\
& \Rightarrow A_{1}, \text { as } n \rightarrow \infty
\end{aligned}
$$

because $\left\{\left(\widetilde{a}_{1.2}-\widehat{a}_{1.2}\right)-E\left(\widetilde{a}_{1.2}-\widehat{a}_{1.2}\right)\right\}$ and $E \widetilde{a}_{1.2}$ are $o_{p}(1)$ by Proposition 4.2 and Lemma 5.4, respectively, of that paper.

Now write $\widetilde{y}_{t}^{+}=y_{t}^{+}+\left(\Omega_{12} \Omega_{22}^{-1}-\widetilde{\Omega}_{12} \widetilde{\Omega}_{22}^{-1}\right) u_{2 t}$, to obtain

$$
\left\|\widetilde{\Pi}_{F D}-\widetilde{\Pi}_{F D}^{+}\right\| \leq\left\|\Omega_{12} \Omega_{22}^{-1}-\widetilde{\Omega}_{12} \widetilde{\Omega}_{22}^{-1}\right\|\left\|\left\{\widehat{F}_{u_{2} x}(1, m)\right\}\left\{\widehat{F}_{x x}(1, m)\right\}^{-1}\right\|
$$

which is $o_{p}(1) O_{p}\left(n^{-1}\right)$, in view of (A.4) and Theorem 5.3 of Robinson and Marinucci (1998); (A.2) follows immediately. 


\section{TABLE I}

Model A: Mean (standard deviation) of $\widetilde{\Pi}-\Pi, n=64, \ell=8, \gamma=1$

$$
\begin{array}{llllll} 
& \rho=.8 & \rho=.4 & \rho=.0 & \rho=-.4 & \rho=-.8 \\
\widetilde{\Pi}_{F M} & -.22(.25) & -.14(.15) & -.13(.13) & -.15(.14) & -.26(.20) \\
\widetilde{\Pi}_{M 0} & -.03(6.3) & .02(.15) & .01(.08) & .00(.05) & .00(.04) \\
\widetilde{\Pi}_{M 1} & -.14(11.3) & .01(.50) & .01(.42) & .00(.05) & .00(.04) \\
\widetilde{\Pi}_{F M}^{*} & -.21(.24) & -.06(.12) & -.03(.08) & -.01(.06) & -.03(.07) \\
\widetilde{\Pi}_{F D} & -.26(.22) & -.08(.11) & -.04(.07) & -.02(.05) & -.01(.04)
\end{array}
$$

Model A: Mean (standard deviation) of $\widetilde{\Pi}-\Pi, n=64, \ell=8, \gamma=0$

$$
\begin{array}{llllll} 
& \rho=.8 & \rho=.4 & \rho=.0 & \rho=-.4 & \rho=-.8 \\
\widetilde{\Pi}_{F M} & .00(.30) & .00(.12) & .00(.08) & .00(.06) & .00(.06) \\
\widetilde{\Pi}_{M 0} & .24(13.0) & .00(.13) & .00(.07) & .00(.05) & .00(.04) \\
\widetilde{\Pi}_{M 1} & .69(40.5) & .00(.17) & .00(.08) & .00(.05) & .00(.04) \\
\widetilde{\Pi}_{F M}^{*} & .01(.30) & .00(.12) & .00(.08) & .00(.05) & .00(.04) \\
\widetilde{\Pi}_{F D} & .01(.28) & .00(.12) & .00(.07) & .00(.05) & .00(.04)
\end{array}
$$




\section{TABLE II}

Model A: Mean (standard deviation) of $\widetilde{\Pi}-\Pi, n=128, \ell=11, \gamma=1$

$$
\begin{array}{llllll} 
& \rho=.8 & \rho=.4 & \rho=.0 & \rho=-.4 & \rho=-.8 \\
\widetilde{\Pi}_{F M} & -.12(.16) & -.07(.09) & -.06(.08) & -.07(.08) & -.15(.15) \\
\widetilde{\Pi}_{M 0} & .05(1.96) & .00(.06) & .00(.03) & .00(.02) & .00(.02) \\
\widetilde{\Pi}_{M 1} & .16(7.3) & .00(.06) & .00(.03) & .00(.02) & .00(.02) \\
\widetilde{\Pi}_{F M}^{*} & -.11(.15) & -.02(.06) & -.01(.04) & -.00(.03) & .01(.03) \\
\widetilde{\Pi}_{F D} & -.14(.14) & -.03(.06) & -.01(.03) & -.01(.03) & .00(.02)
\end{array}
$$

Model A: Mean (standard deviation) of $\widetilde{\Pi}-\Pi, n=128, \ell=11, \gamma=0$

$$
\begin{array}{llllll} 
& \rho=.8 & \rho=.4 & \rho=.0 & \rho=-.4 & \rho=-.8 \\
\widetilde{\Pi}_{F M} & .00(.17) & .00(.06) & .00(.04) & .00(.03) & .00(.02) \\
\widetilde{\Pi}_{M 0} & .02(1.12) & .00(.06) & .00(.03) & .00(.02) & .00(.02) \\
\widetilde{\Pi}_{M 1} & .00(.62) & .00(.06) & .00(.03) & .00(.02) & .00(.02) \\
\widetilde{\Pi}_{F M}^{*} & .00(.16) & .00(.06) & .00(.04) & .00(.03) & .00(.02) \\
\widetilde{\Pi}_{F D} & .00(.16) & .00(.06) & .00(.03) & .00(.02) & .00(.02)
\end{array}
$$




\section{TABLE III}

Model A: Type I error at $5 \%$ of $\chi_{\widetilde{\Pi}-\Pi}^{2}, n=64, \ell=8, \gamma=1$

$$
\begin{array}{llllll} 
& \rho=.8 & \rho=.4 & \rho=.0 & \rho=-.4 & \rho=-.8 \\
\widetilde{\Pi}_{F M} & 32.60 \% & 24.34 \% & 25.46 \% & 27.46 \% & 36.82 \% \\
\widetilde{\Pi}_{M 0} & 37.86 \% & 17.60 \% & 13.36 \% & 11.44 \% & 15.60 \% \\
\widetilde{\Pi}_{M 1} & 41.32 \% & 19.72 \% & 15.00 \% & 12.46 \% & 11.84 \% \\
\widetilde{\Pi}_{F M}^{*} & 31.48 \% & 15.42 \% & 12.86 \% & 11.48 \% & 30.06 \% \\
\widetilde{\Pi}_{F D} & 36.90 \% & 18.78 \% & 13.02 \% & 9.84 \% & 10.58 \%
\end{array}
$$

Model A: Type I error at $5 \%$ of $\chi_{\widetilde{\Pi}-\Pi}^{2}, n=64, \ell=8, \gamma=0$

$\begin{array}{llllll} & \rho=.8 & \rho=.4 & \rho=.0 & \rho=-.4 & \rho=-.8 \\ \widetilde{\Pi}_{F M} & 16.56 \% & 11.56 \% & 9.72 \% & 8.94 \% & 7.78 \% \\ \widetilde{\Pi}_{M 0} & 32.82 \% & 13.16 \% & 8.82 \% & 7.66 \% & 7.18 \% \\ \widetilde{\Pi}_{M 1} & 37.10 \% & 16.54 \% & 11.78 \% & 10.72 \% & 10.00 \% \\ \widetilde{\Pi}_{F M}^{*} & 14.64 \% & 8.58 \% & 6.96 \% & 5.74 \% & 2.76 \% \\ \widetilde{\Pi}_{F D} & 12.90 \% & 7.78 \% & 6.40 \% & 4.98 \% & 2.22 \%\end{array}$




\section{TABLE IV}

Model A: Type I error at $5 \%$ of $\chi_{\widetilde{\Pi}-\Pi}^{2}, n=128, \ell=11, \gamma=1$

$$
\begin{array}{llllll} 
& \rho=.8 & \rho=.4 & \rho=.0 & \rho=-.4 & \rho=-.8 \\
\widetilde{\Pi}_{F M} & 25.72 \% & 19.96 \% & 17.68 \% & 20.34 \% & 31.88 \% \\
\widetilde{\Pi}_{M 0} & 23.24 \% & 10.74 \% & 8.12 \% & 8.16 \% & 10.40 \% \\
\widetilde{\Pi}_{M 1} & 24.92 \% & 11.82 \% & 9.28 \% & 8.36 \% & 8.18 \% \\
\widetilde{\Pi}_{F M}^{*} & 24.30 \% & 10.26 \% & 8.06 \% & 7.60 \% & 22.20 \% \\
\widetilde{\Pi}_{F D} & 30.50 \% & 11.78 \% & 7.98 \% & 6.52 \% & 7.34 \%
\end{array}
$$

Model A: Type I error at $5 \%$ of $\chi_{\widetilde{\Pi}-\Pi}^{2}, n=128, \ell=11, \gamma=0$

$\begin{array}{llllll} & \rho=.8 & \rho=.4 & \rho=.0 & \rho=-.4 & \rho=-.8 \\ \widetilde{\Pi}_{F M} & 13.56 \% & 9.98 \% & 8.92 \% & 7.84 \% & 6.20 \% \\ \widetilde{\Pi}_{M 0} & 18.50 \% & 9.02 \% & 6.92 \% & 5.96 \% & 5.90 \% \\ \widetilde{\Pi}_{M 1} & 20.02 \% & 10.72 \% & 8.18 \% & 7.38 \% & 7.26 \% \\ \widetilde{\Pi}_{F M}^{*} & 10.94 \% & 7.62 \% & 5.98 \% & 5.26 \% & 2.78 \% \\ \widetilde{\Pi}_{F D} & 10.28 \% & 6.92 \% & 5.24 \% & 4.90 \% & 2.42 \%\end{array}$




\section{TABLE V}

Model B: Mean (standard deviation) of $\widetilde{\Pi}-\Pi, n=64, \ell=8, \gamma=1$

$$
\begin{array}{lllll} 
& \rho_{1}=.947 & \rho_{1}=.34 & \rho_{1}=-.34 & \rho_{1}=-.947 \\
\widetilde{\Pi}_{F M} & -. .33(.24) & -.46(.30) & -.30(.22) & -.50(.37) \\
\widetilde{\Pi}_{M 0} & .02(.47) & .00(.07) & .00(.04) & .00(.03) \\
\widetilde{\Pi}_{M 1} & .01(.08) & .00(.05) & .00(.03) & .00(.02) \\
\widetilde{\Pi}_{F M}^{*} & -.07(.09) & .03(.08) & -.08(.08) & .10(.09) \\
\widetilde{\Pi}_{F D} & -.05(.08) & -.02(.05) & -.01(.04) & .00(.03)
\end{array}
$$

Model B: Mean (standard deviation) of $\widetilde{\Pi}-\Pi, n=64, \ell=8, \gamma=0$

$$
\begin{array}{lcccc} 
& \rho_{1}=.947 & \rho_{1}=.34 & \rho_{1}=-.34 & \rho_{1}=-.947 \\
\widetilde{\Pi}_{F M} & .00(.11) & .00(.11) & .00(.06) & .00(.08) \\
\widetilde{\Pi}_{M 0} & .02(1.3) & .00(.06) & .00(.04) & .00(.03) \\
\widetilde{\Pi}_{M 1} & .00(.07) & .00(.04) & .00(.03) & .00(.02) \\
\widetilde{\Pi}_{F M}^{*} & .00(.09) & .00(.05) & .00(.04) & .00(.03) \\
\widetilde{\Pi}_{F D} & .00(.08) & .00(.05) & .00(.03) & .00(.03)
\end{array}
$$




\section{TABLE VI}

Model B: Mean (standard deviation) of $\widetilde{\Pi}-\Pi, n=128, \ell=11, \gamma=1$

$$
\begin{array}{lllll} 
& \rho_{1}=.947 & \rho_{1}=.34 & \rho_{1}=-.34 & \rho_{1}=-.947 \\
\widetilde{\Pi}_{F M} & -.36(.23) & -.17(.17) & -.21(.18) & -.35(.29) \\
\widetilde{\Pi}_{M 0} & .00(.05) & .00(.03) & .00(.02) & .00(.01) \\
\widetilde{\Pi}_{M 1} & .00(.04) & .00(.02) & .00(.01) & .00(.01) \\
\widetilde{\Pi}_{F M}^{*} & .02(.05) & .03(.04) & -.08(.07) & .07(.06) \\
\widetilde{\Pi}_{F D} & -.01(.04) & -.00(.02) & .00(.02) & .00(.01)
\end{array}
$$

Model B: Mean (standard deviation) of $\widetilde{\Pi}-\Pi, n=128, \ell=11, \gamma=0$

$$
\begin{array}{lllll} 
& \rho_{1}=.947 & \rho_{1}=.34 & \rho_{1}=-.34 & \rho_{1}=-.947 \\
\widetilde{\Pi}_{F M} & .00(.06) & .00(.03) & .00(.03) & .00(.03) \\
\widetilde{\Pi}_{M 0} & .00(.05) & .00(.03) & .00(.02) & .00(.01) \\
\widetilde{\Pi}_{M 1} & .00(.04) & .00(.02) & .00(.01) & .00(.01) \\
\widetilde{\Pi}_{F M}^{*} & .00(.04) & .00(.02) & .00(.02) & .00(.01) \\
\widetilde{\Pi}_{F D} & .00(.04) & .00(.02) & .00(.02) & .00(.01)
\end{array}
$$




\section{TABLE VII}

Model B: Type I error at $5 \%$ of $\chi_{\widetilde{\Pi}-\Pi}^{2}, n=64, \ell=8, \gamma=1$

$$
\begin{array}{rllll} 
& \rho_{1}=.947 & \rho_{1}=.34 & \rho_{1}=-.34 & \rho_{1}=-.947 \\
\widetilde{\Pi}_{F M} & 39.4 \% & 61.92 \% & 35.30 \% & 63.64 \% \\
\widetilde{\Pi}_{M 0} & 2.82 \% & 0.62 \% & .12 \% & .30 \% \\
\widetilde{\Pi}_{M 1} & 21.04 \% & 16.96 \% & 14.96 \% & 23.42 \% \\
\widetilde{\Pi}_{F M}^{*} & 22.48 \% & 7.71 \% & 52.74 \% & 74.98 \% \\
\widetilde{\Pi}_{F D} & 13.92 \% & 5.02 \% & 5.18 \% & 10.52 \%
\end{array}
$$

Model B: Type I error at $5 \%$ of $\chi_{\widetilde{\Pi}-\Pi}^{2}, n=64, \ell=8, \gamma=0$

$\begin{array}{lllll} & \rho_{1}=.947 & \rho_{1}=.34 & \rho_{1}=-.34 & \rho_{1}=-.947 \\ \widetilde{\Pi}_{F M} & 3.08 \% & 13.10 \% & 2.22 \% & 13.78 \% \\ \widetilde{\Pi}_{M 0} & .46 \% & .00 \% & .02 \% & .00 \% \\ \widetilde{\Pi}_{M 1} & 7.48 \% & 7.48 \% & 7.56 \% & 7.10 \% \\ \widetilde{\Pi}_{F M}^{*} & 1.02 \% & 5.24 \% & .34 \% & 1.18 \% \\ \widetilde{\Pi}_{F D} & 6.80 \% & 4.95 \% & .08 \% & .44 \%\end{array}$




\section{TABLE VIII}

Model B: Type I error at $5 \%$ of $\chi_{\widetilde{\Pi}-\Pi}^{2}, n=128, \ell=11, \gamma=1$

$\begin{array}{lllll} & \rho_{1}=.947 & \rho_{1}=.34 & \rho_{1}=-.34 & \rho_{1}=-.947 \\ \widetilde{\Pi}_{F M} & 61.34 \% & 27.56 \% & 31.50 \% & 59.70 \% \\ \widetilde{\Pi}_{M 0} & .08 \% & .02 \% & .00 \% & .00 \% \\ \widetilde{\Pi}_{M 1} & 14.88 \% & 10.12 \% & 9.88 \% & 8.72 \% \\ \widetilde{\Pi}_{F M}^{*} & 22.78 \% & 33.32 \% & 76.82 \% & 83.24 \% \\ \widetilde{\Pi}_{F D} & 10.66 \% & 3.22 \% & 3.22 \% & 5.84 \%\end{array}$

Model B: Type I error at $5 \%$ of $\chi_{\widetilde{\Pi}-\Pi}^{2}, n=128, \ell=11, \gamma=0$

$\begin{array}{lllll} & \rho_{1}=.947 & \rho_{1}=.34 & \rho_{1}=-.34 & \rho_{1}=-.947 \\ \widetilde{\Pi}_{F M} & 9.08 \% & 2.82 \% & 2.48 \% & 7.96 \% \\ \widetilde{\Pi}_{M 0} & .00 \% & .00 \% & .00 \% & .00 \% \\ \widetilde{\Pi}_{M 1} & 7.00 \% & 6.30 \% & 6.18 \% & 1.18 \% \\ \widetilde{\Pi}_{F M}^{*} & 2.60 \% & .66 \% & .36 \% & .76 \% \\ \widetilde{\Pi}_{F D} & 1.90 \% & .36 \% & .20 \% & .26 \%\end{array}$

